this analysis of longitudinal ANA/autoantibody profiles in relation to SLE outcomes, suggesting these subsets might be identifiable based on extended autoantibody profiles early in disease and carry prognostic information.

Acknowledgments This study is presented on behalf of SLICC. We would also like to acknowledge the technical assistance of Ms. Haiyan Hou (MitogenDx Laboratory).

\section{PROTEIN ASSEMBLAGES ARE NEWLY DESCRIBED INTRACELLULAR STRUCTURES THAT MAY PLAY A ROLE IN SHAPING THE LUPUS AUTOANTIBODY REPERTOIRE}

'Philip L Carl, ${ }^{2}$ Howard M Fried, ${ }^{3}$ Philip L Cohen*. 'Department of Pharmacology, University of North Carolina at Chapel Hill; ${ }^{2}$ Department of Biochemistry and Biophysics, University of North Carolina at Chapel Hill; ${ }^{3}$ Section of Rheumatology, Department of Medicine, Lewis Katz School of Medicine at Temple University, USA

\subsection{6/lupus-2021-lupus21 century. 100}

Background Why are autoantibodies in systemic autoimmunity directed against only $\sim 5 \%$ of the proteome? It has recently been discovered that many intracellular proteins, rather than being evenly distributed throughout the cell, instead form assemblages (also known as Membraneless Organelles and Biological Condensates) that arise from phase separation of their protein components, akin to partitioning of oil droplets in water. Such a conformation might be potentially more immunogenic than that of proteins with a diffuse presence in cells. We wondered if lupus autoantigens might preferentially exist as assemblages and thereby provoke autoantibody production.

Methods We obtained from an assemblage prediction algorithm (Vernon et al., elife 7, 2018) the propensity scores (PScores), i.e., likelihood, for phase separation of autoantigens and non-autoantigens. We then compared autoantigens with the highest PScores to identify shared structural properties. We used the European Molecular Biology Laboratory 'InTact' Molecular Interactions Database to assess the potential for interactions of autoantigens compared with nonautoantigens.

Results The mean PScores for autoantigens ( $\mathrm{n}=1050)$ and the entire human proteome of non-autoantigens $(\mathrm{n}=17,532)$ were 1.46 and 1.09 ( $\mathrm{p}=1.2 \mathrm{E}-08$ ). To varying extents, the 25 autoantigens with the highest phase separation propensities shared additional features such as compositional bias, repeated domains, coiled coil regions, nucleic acid binding, and disorder. Most of these properties were present with greater frequencies than observed for non-autoantigens. When potential interactions were compared using InTact, autoantigens had at least a ten-fold greater tendency to interact with themselves and other proteins.

Conclusions We suggest that assemblage formation and certain protein structural features are key factors in determining the spectrum of lupus autoantibodies. Assemblages may promote autoantibody formation by concentrating certain intracellular proteins, as concentrations may be $\sim 100 \mathrm{x}$ or more that of the intracellular proteins not contained in assemblages or organelles. Assemblages also foster protein-protein interactions, which could lead to changes in conformation of one or both partners, resulting in novel conformations to which the immune system might not be tolerized.

\section{6 \\ A MODEL OF LUPUS PATHOGENESIS: ANTI-EBNA1 HETEROANTIBODIES INITIATE LUPUS BY CROSS REACTING WITH LUPUS AUTOANTIGENS, RESULTING IN LUPUS AUTOANTIBODIES AND CLINICAL DISEASE}

${ }^{1}$ Viktoryia Laurynenka, ${ }^{2,3}$ Lili Ding, 1,3 Leah C Kottyan, 1,3 Matthew T Weirauch, 1,3,4 Kenneth M Kaufman, ${ }^{5}$ Judith A James, ${ }^{4}$ John B Harley*. ${ }^{1}$ Center for Autoimmune Genomics and Etiology, Cincinnati Children's Hospital Medical Center, Cincinnati, Ohio, USA; ${ }^{2}$ Division of Biostatistics and Epidemiology, Department of Pediatrics, Cincinnati Children's Hospital Medical Center, Cincinnati, Ohio, USA; ${ }^{3}$ University of Cincinnati College of Medicine, Cincinnati, Ohio, USA; ${ }^{4}$ Research Service, US Department of Veterans Affairs (USDVA) Medical Center, Cincinnati, Ohio, USA; ${ }^{5}$ Arthritis and Clinical Immunology Program, Oklahoma Medical Research Foundation and the University of Oklahoma Health Sciences Center, Oklahoma City, Oklahoma, USA

\subsection{6/lupus-2021-lupus21century. 101}

Background Mechanisms explaining the well-known association of SLE with Epstein-Barr virus (EBV) infection are unknown. In other experiments, the Epstein-Barr nuclear antigen-2, -3C, \& -LP (EBNA2, EBNA3C, EBNALP), all EBV-encoded transcription co-factors, have been shown to concentrate at SLE genetic risk loci, supporting the hypothesis that the host immune response to EBV initiates SLE autoimmune processes. Cross-reactions of the anti-EBNA1 heteroimmune response with the Sm B/B', Sm D, Ro/SSA, and C1q autoantigens are convincing. Some of these cross reacting EBNA1 epitopes appear to be the initiating the autoantibodies in SLE patients and induce SLE-like disease in animals after immunization. EBNA1 aberrantly reduces $\mathrm{T}$ cell responses in normal individuals. Perhaps, the first SLE autoantibodies usually emerge from the anti-EBNA1 humoral response. If this hypothesis is the common sequence of events in SLE, then anti-EBNA1 responses would be predicted to be more frequent in EBVinfected SLE patients than in EBV-infected controls.

Methods We tested this prediction using the large dataset in East Asians published by Cui, et al (PLoS One 2018;13: e01931711) by matching controls to cases by age and sex and using conditional logistic regression.

Results All 232 SLE patients (100\%) were EBV-infected, while 54 of 696 patient controls were not EBV infected (7.6\%), resulting in a strong association $\left(\mathrm{OR}=28.6\left(6.4-\infty, \mathrm{p}=5 \times 10^{-8}\right)\right.$, further confirming the known close association of EBV infection with SLE with a $100 \%$ attributable fraction. Most importantly, virtually all the SLE cases tested for both anti-VCA IgG and anti-EBNA1 IgG also had anti-EBNA1 antibodies (124 of 125 (99.2\%)), which were more frequent than in age- and sex-matched controls (232 of 250 (93.2\%)) (OR=9.7, 95\%CI $1.5-414, p=0.0078$ ) for an $89.7 \%$ additional attributable fraction among those EBV-infected, thereby adding anti-EBNA1 antibodies as an SLE risk factor beyond EBV infection.

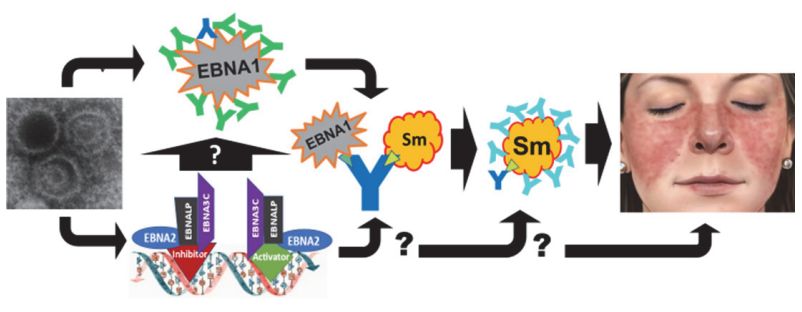

Images courtesy of NIAID (left) \& Mayo Foundation (right), all rights reserved

Abstract 1706 Figure 1 
Conclusions These data (Cui, et al. PLoS One 2018;13: e01931711), also show strong association of EBV with SLE with anti-EBNA1 being more frequent in SLE cases than in concurrent patient controls, supporting the known association of EBV infection with SLE. Further, anti-EBNA1 is virtually always present in SLE and is present more frequently in EBA-infected SLE patients than in EBV-infected controls, consistent with the hypothesis that anti-EBNA1 is the usual immune response foundation from which pathogenic SLE autoimmunity emerges. These results support a model of the etiology of SLE that begins with EBV infection, leading to anti-EBNA1 antibodies. Then, a subset of these heteroimmune anti-EBNA1 antibodies cross react with SLE autoantigens (e.g., $\mathrm{Sm}$ as in figure 1, (EBV image, courtesy of NIAID/NIH; SLE malar rash image, courtesy of Mayo Foundation, all rights reserved)) that then mature into an SLE autoantibody response and result in in life-threatening clinical SLE. The specific role of the other EBV gene products, EBNA2, EBNA3C, and EBNALP, in this process (figure 1) await elucidation.

Acknowledgments Support is appreciated from US Department of Veterans Affairs Merit Award (I01 BX001834), and the National Institutes of Health (R01 AI24717 \& $\quad$ U01 AI130830).

\section{ANTI-RNP ANTIBODIES ARE ASSOCIATED WITH THE INTERFERON GENE SIGNATURE BUT NOT COMPLEMENT ACTIVATION IN SLE}

${ }^{1}$ Erika Hubbard*, ${ }^{2,3}$ David S Pisetsky, ${ }^{1}$ Peter Lipsky. 'AMPEL BioSolutions, LLC and RILITE Foundation, Charlottesville, VA, USA; ${ }^{2}$ Duke University Medical Center, Durham, NC, USA; ${ }^{3}$ VA Medical Center, Durham, NC, USA

10.1136/lupus-2021-lupus21century. 102

Background Anti-nuclear antibodies are essential features of SLE and are important markers for both diagnosis and pathogenesis. Anti-double stranded DNA (dsDNA) antibodies, which are routinely monitored to assess disease activity, can form immune complexes that activate complement (C) and promote renal pathology. Relatively less is known about the roles of autoantibodies against RNA binding proteins (RBPs) in pathogenesis and about the relationship between antidsDNA, anti-RBPs, complement activation, and expression of the interferon (IFN) gene signature (IGS). Analysis of data from two clinical trials of tabalumab in SLE (Illuminate $1 \&$ 2, GSE88884) was undertaken to understand these interrelationships.

Methods Microarray data from 1620 active (SLEDAI $\geq 6$ ), female SLE patients and accompanying laboratory measurements were analyzed. Gene set variation analysis (GSVA) determined enrichment of transcriptomic signatures in each patient. Linear regression analysis was used to determine relationships between IGS GSVA scores and C3 and/or C4 levels as well as autoantibody levels. Unbiased classification and regression trees (CART) were constructed to determine the highest predictors of IGS expression.

Results Patients were initially stratified by autoantibody positivity. Comparison of GSVA enrichment scores of a common IGS demonstrated that SLE patients positive for anti-RNP antibodies alone had greater enrichment of the IGS than those positive for anti-dsDNA alone. Similar results were noted for gene signatures of type I IFN, IFN $\alpha 2$, IFN $\beta$, and IFN $\gamma$. In contrast, the TNF-induced gene signature was observed comparably in patients with anti-dsDNA or anti-RNP antibodies and an IL-1 gene signature was only observed in those with anti-dsDNA. By linear regression, IFN GSVA scores and C3 and C4 levels were significantly, inversely related in antidsDNA+ patients but not those with anti-RNP antibodies. Antibody levels correlated with decreased C levels in dsDNA+ patients. Additionally, IGS GSVA scores were increased in anti-dsDNA+ patients with low C3 or C4 compared to antidsDNA+ patients with high/normal C, but there was no significant difference in IGS expression in anti-RNP+ patients with the same stratification. CART analysis identified anti-RNP status as the highest predictor of the IGS GSVA score. Finally, $54.5 \%$ of SLE patients without IGS expression exhibited autoantibodies, but only $13.7 \%$ of SLE patients negative for autoantibodies expressed the IGS signature.

Conclusions Taken together, these data indicate that anti-RNP antibodies are associated with IGS expression more strongly than anti-dsDNA antibodies but are not related to the depression of C3 or C4. Furthermore, IGS expression is not required for autoantibody production, but autoantibodies are likely directionally related to the IGS signature.

\section{PRELIMINARY DATA ON THE MAPPING OF ANTI- MITOCHONDRIAL ANTIBODIES IN SYSTEMIC LUPUS ERYTHEMATOSUS}

\begin{abstract}
1,2 Yann LC Becker*, 1,2 Emmanuelle Rollet-Labelle, ${ }^{1,2}$ Tania Lévesque, ${ }^{1,2}$ Joannie Leclerc, ${ }^{3}$ Anne-Sophie Julien, ${ }^{1,4}$ Paul R Fortin, SLICC inception cohort investigators, 1,2Éric Boilard. ${ }^{1}$ Centre de Recherche ARThrite - Arthrite, Recherche et Traitements, Université Laval, Québec, QC, Canada; ${ }^{2}$ Centre de Recherche du CHU de Québec - Université Laval, Québec, QC, Canada; ${ }^{3}$ Département de mathématiques et statistique, Université Laval, Québec, QC, Canada; ${ }^{4}$ Division de Rhumatologie, Département de Médecine, CHU de Québec Université Laval, Québec, QC, Canada
\end{abstract}

10.1136/lupus-2021-lupus21 century. 103

Background In systemic lupus erythematosus (SLE), mitochondria and their inner components may be released into the extracellular space, potentially eliciting a pro-inflammatory response by the immune system. While cardiolipin was long known as a mitochondrial target of autoantibodies, we reported in previous case-control studies that autoantibodies to whole mitochondria (AwMA), mitochondrial DNA (AmtDNA) and mitochondrial RNA (AmtRNA) are also targeted by SLE autoantibodies. We aim to characterize levels of these autoantibodies throughout SLE disease progression.

Methods Anti-mitochondrial antibodies (AMA, IgGs) targeting whole mitochondria (AwMA), mtDNA (AmtDNA) or mtRNA (AmtRNA) were measured by direct-ELISA, using sera from the Systemic Lupus International Collaborating Clinics (SLICC) inception cohort. Samples comprised healthy controls $(n=127)$ and 3453 samples obtained from 816 SLE patients, between the diagnosis up to 7 years afterward. Institution of the SLICC cohort obtained approval from their local research ethic boards and written consent from every participant. Eligibility to the cohort, within 15 months of diagnosis, was conditional to the positivity to 4 , or more, ACR criteria for the classification of SLE. AMA levels are expressed as the median optical density measured at $405 \mathrm{~nm} \pm$ interquartile range. Differences in AMA levels between healthy donors and baseline SLE samples were assessed, using Mann-Whitney tests and 